EPJ Web of Conferences 59, 05021 (2013)

DOI: $10.1051 /$ epjconf/20135905021

(C) Owned by the authors, published by EDP Sciences, 2013

\title{
Kinetic model for energy deposition in fast ignition
}

\author{
S.Z. Wu ${ }^{a}$, H. Zhang ${ }^{\text {b }}$, C.T. Zhou, S.P. Zhu and X.T. He \\ Institute of Applied Physics and Computational Mathematics, PO Box 8009, Beijing 100088, \\ PR China
}

\begin{abstract}
A kinetic model describing the energy deposition of fast electrons is established by considering both binary collisions and contributions due to collective process. The collision term is exactly simplified by taking into account relativistic effects within the context of fast ignition, and the explicit formulation of relativistic kinetic equation in three-dimensional momentum space is obtained with the help of spherical harmonic expansion. The corresponding numerical code is developed and benchmarked physically. Typical case studies in fast ignition are also presented.
\end{abstract}

\section{INTRODUCTION}

Fast ignition (FI) [1] presents an alternative approach to realize inertial confinement fusion (ICF), in which hot spot formation is separated from capsule compression. It has great potential advantages over conventional ICF, notably in relaxing the requirements of implosion symmetry and hydrodynamic instabilities, leading to higher gains with lower driver energy, which has drawn significant attention worldwide [2-9]. In FI scheme, fast electrons generated via ultraintense $\left(\sim 10^{20} \mathrm{~W} / \mathrm{cm}^{2}\right)$ short-pulse $(\sim 20 \mathrm{ps})$ laser interaction with plasmas will transport into the pre-compressed dense core to deposit energy locally, forming the hot spot. Therefore, it is of great importance to study this process which will ultimately determine the size and temperature of the hot spot, and hence the thermonuclear burn dynamics.

In this paper, a kinetic model to describe the energy loss of fast electrons is proposed. The relevant numerical code based on the kinetic equation is developed and verified. Several typical case studies involved in FI are performed and show that the established kinetic model is of practical use.

\section{KINETIC MODEL AND EQUATION}

Fast electrons incident into highly overdense core plasmas, the energy loss and deposition to the background is mainly due to collisions $[1,2,4]$. The collisions between fast electrons and background particles generally fall into two types, namely, the short-range and the long-rang collisions, depending on the impact parameter $l$. The former refers to the binary Coulomb collisions with impact parameter less than Debye length $\left(l<\lambda_{D}\right)$, which is well described by Fokker-Planck (FP) collision operator. The latter is associated with the collective response of target plasmas to the fast electrons $\left(l \geq \lambda_{D}\right)$, which is often treated with Balescu-Lenard (BL) collision term. The collisions between the fast electrons will not be considered here, since the beam density is far less than the target density in FI context.

\footnotetext{
${ }^{a}$ e-mail: wu_sizhong@iapcm.ac.cn

be-mail: zhang_hua@iapcm.ac.cn
}

This is an Open Access article distributed under the terms of the Creative Commons Attribution License 2.0, which permits unrestricted use, distribution, and reproduction in any medium, provided the original work is properly cited. 


\section{EPJ Web of Conferences}

The relativistic kinetic equation with collisions for fast electrons can be expressed as [10]

$$
\frac{\partial f_{e}}{\partial t}+\frac{\mathbf{p}}{\gamma m} \cdot \nabla f_{e}-e\left(\mathbf{E}+\frac{\mathbf{p} \times \mathbf{B}}{\gamma m}\right) \cdot \nabla_{\mathbf{p}} f_{e}=\sum_{b=b e, b i} \nabla_{\mathbf{p}} \cdot \int Q \cdot\left[f_{b} \nabla_{\mathbf{p}} f_{e}-f_{e} \nabla_{\mathbf{p}^{\prime}} f_{b}\right] d^{3} \mathbf{p}^{\prime},
$$

where subscripts $e$ and $b$ refer to fast electrons and background plasma species (be for background electron and $b i$ for background ion). $f_{e}$ (or $f_{b}$ ) denotes the distribution function for $e$ (or species $b$ ). $\mathbf{E}$ and $\mathbf{B}$ are the electric and magnetic fields. p, $\gamma$ and $m$ represent the momentum, the relativistic Lorentz factor and the rest mass of fast electrons, respectively. The physical quantities for background plasma species $b$ are all labeled with superscript ' throughout this paper. $Q$ is the collision kernel, and it can be casted into two parts as mentioned before. $Q=Q_{\mathrm{FP}}+Q_{\mathrm{BL}} \cdot Q_{\mathrm{FP}}=2 \pi e^{2} q^{\prime 2} \ln \Lambda_{\mathrm{FP}} \frac{u^{2} \mathbf{I}-\mathbf{u u}}{u^{3}}$ with $\ln \Lambda_{\mathrm{FP}}=$ $\ln \left[\Lambda_{D}^{2} m^{2} c^{2}(\gamma-1) / 2 \hbar^{2}\right]+1+(1-1 / \gamma)^{2} / 8-(2 \gamma-1) \ln 2 / \gamma^{2}$, and $Q_{\mathrm{BL}}=2 \pi e^{2} q^{\prime 2} \ln \Lambda_{\mathrm{BL}} \frac{u^{2} \mathbf{I}-\mathbf{u u}}{u^{3}}$ with $\ln \Lambda_{\mathrm{BL}}=\ln |A-1| / 2-A / 2(A-1), A=2 p^{2} / 3 \gamma^{2} \Lambda_{D}^{2} \omega_{p}^{2}$. Here, $\mathbf{u}=\mathbf{v}-\mathbf{v}^{\prime}$ is the relative velocity of the two colliding particles. Then the right hand side (labelled as $R H S$ ) of Eq. (1) can be simplified,

$$
R H S=\Gamma \nabla_{\mathbf{p}} \cdot\left[\left(\nabla_{\mathbf{v}} \nabla_{\mathbf{v}} g\right) \cdot \nabla_{\mathbf{p}} f_{e}-\frac{m}{m^{\prime}} f_{e} \nabla_{\mathbf{v}}\left(\gamma \nabla_{\mathbf{p}} \cdot \nabla_{\mathbf{v}} h\right)\right],
$$

where $\Gamma=2 \pi e^{2} q^{\prime 2} \ln \Lambda \quad\left(\ln \Lambda=\ln \Lambda_{\mathrm{FP}}+\ln \Lambda_{\mathrm{BL}} \quad\right.$ represents the Coulomb logarithm), and $g=$ $\int u f_{b} d^{3} \mathbf{p}^{\prime}, h=\int \frac{u}{\gamma^{\prime}} f_{b} d^{3} \mathbf{p}^{\prime}$. By expanding $f_{b}$ in terms of spherical harmonics $Y_{l k}, f_{b}\left(\mathbf{p}^{\prime}\right)=$ $\sum_{l=0}^{\infty} \sum_{k=-l}^{l} F_{l k}\left(p^{\prime}\right) Y_{l k}\left(\theta^{\prime}, \phi^{\prime}\right)$, the expressions for $g$ and $h$ can be explicitly deduced and substituted into Eq. (2), leading to the final form of kinetic equation in three-dimensional (3D) momentum space,

$$
\begin{aligned}
& {\left[\frac{\partial}{\partial t}+\frac{\mathbf{p}}{\gamma m} \cdot \nabla-e\left(\mathbf{E}+\frac{\mathbf{p} \times \mathbf{B}}{\gamma m}\right) \cdot \nabla_{\mathbf{p}}\right] f_{e}=\sum_{b=b e, b i}\left[\frac{\Gamma}{p^{2}} \frac{\partial}{\partial p}\left(A_{1}+A_{2} \frac{\partial}{\partial p}+A_{3} \frac{\partial}{\partial \theta}+A_{4} \frac{\partial}{\partial \phi}\right) f_{e}\right.} \\
& \left.+\frac{\Gamma}{p^{2} \sin \theta} \frac{\partial}{\partial \theta}\left(B_{1}+B_{2} \frac{\partial}{\partial p}+B_{3} \frac{\partial}{\partial \theta}+B_{4} \frac{\partial}{\partial \phi}\right) f_{e}+\frac{\Gamma}{p^{2} \sin \theta} \frac{\partial}{\partial \phi}\left(C_{1}+C_{2} \frac{\partial}{\partial p}+C_{3} \frac{\partial}{\partial \theta}+C_{4} \frac{\partial}{\partial \phi}\right) f_{e}\right] .
\end{aligned}
$$

with the coefficients

$$
\begin{aligned}
& A_{1}=-\frac{m^{2}}{m^{\prime}} \sum_{l=0}^{\infty} \sum_{k=-l}^{l}\left\{v^{2} \partial_{v}^{3} H_{l k}+2 v \partial_{v}^{2} H_{l k}-\gamma^{2}[2+l(l+1)] \partial_{v} H_{l k}+\frac{2 \gamma^{2}}{v} l(l+1) H_{l k}\right\} Y_{l k}, \\
& A_{2}=\sum_{l=0}^{\infty} \sum_{k=-l}^{l} p^{2}\left(\partial_{v}^{2} G_{l k}\right) Y_{l k}, \\
& A_{3}=\sum_{l=0}^{\infty} \sum_{k=-l}^{l}\left[\gamma m\left(\partial_{v} G_{l k}\right)-\frac{\gamma^{2} m^{2} G_{l k}}{p}\right]\left(\partial_{\theta} Y_{l k}\right), \\
& A_{4}=\sum_{l=0}^{\infty} \sum_{k=-l}^{l} \frac{1}{\sin ^{2} \theta}\left[\gamma m\left(\partial_{v} G_{l k}\right)-\frac{\gamma^{2} m^{2} G_{l k}}{p^{2}}\right]\left(\partial_{\theta} Y_{l k}\right), \\
& B_{1}=-\frac{m}{m^{\prime}} \gamma \sin \theta \sum_{l=0}^{\infty} \sum_{k=-l}^{l}\left[\frac{\partial_{v}^{2} H_{l k}}{\gamma^{2}}+\frac{2 \partial_{v} H_{l k}}{v}-\frac{l(l+1) H_{l k}}{v^{2}}\right]\left(\partial_{\theta} Y_{l k}\right),
\end{aligned}
$$




$$
\begin{aligned}
B_{2} & =\sum_{l=0}^{\infty} \sum_{k=-l}^{l} \sin \theta\left[\gamma m\left(\partial_{v} G_{l k}\right)-\gamma^{2} m^{2} \frac{G_{l k}}{p}\right]\left(\partial_{\theta} Y_{l k}\right), \\
B_{3} & =\sum_{l=0}^{\infty} \sum_{k=-l}^{l} \sin \theta\left[\frac{\gamma m\left(\partial_{v} G_{l k}\right)}{p} Y_{l k}+\frac{\gamma^{2} m^{2} G_{l k}}{p^{2}}\left(\partial_{\theta}^{2} Y_{l k}\right)\right], \\
B_{4} & =\sum_{l=0}^{\infty} \sum_{k=-l}^{l} \frac{\gamma^{2} m^{2} G_{l k}}{p^{2}}\left[\frac{1}{\sin \theta}\left(\partial_{\theta \phi}^{2} Y_{l k}\right)-\frac{\cos \theta}{\sin ^{2} \theta}\left(\partial_{\phi} Y_{l k}\right)\right], \\
C_{1} & =-\frac{m}{m^{\prime}} \frac{\gamma}{\sin \theta} \sum_{l=0}^{\infty} \sum_{k=-l}^{l}\left[\frac{1}{\gamma^{2}} \partial_{v}^{2} H_{l k}+\frac{2}{v} \partial_{v} H_{l k}-\frac{l(l+1)}{v^{2}} H_{l k}\right]\left(\partial_{\phi} Y_{l k}\right), \\
C_{2} & =\sum_{l=0}^{\infty} \sum_{k=-l}^{l} \frac{1}{\sin \theta}\left[\gamma m\left(\partial_{v} G_{l k}\right)-\gamma^{2} m^{2} \frac{G_{l k}}{p}\right]\left(\partial_{\phi} Y_{l k}\right), \\
C_{3}= & \sum_{l=0}^{\infty} \sum_{k=-l}^{l} \frac{\gamma^{2} m^{2} G_{l k}}{p^{2}}\left[\frac{\partial_{\theta \phi}^{2} Y_{l k}}{\sin \theta}-\frac{\cos \theta\left(\partial_{\phi} Y_{l k}\right)}{\sin ^{2} \theta}\right], \\
C_{4}= & \sum_{l=0}^{\infty} \sum_{k=-l}^{l} \frac{1}{\sin \theta}\left[\frac{\gamma m\left(\partial_{v} G_{l k}\right)}{p} Y_{l k}+\frac{\gamma^{2} m^{2} G_{l k}}{p^{2}} \cot \theta\left(\partial_{\theta} Y_{l k}\right)+\frac{\gamma^{2} m^{2}\left(\partial_{\phi}^{2} Y_{l k}\right)}{p^{2} \sin ^{2} \theta}\right] .
\end{aligned}
$$

The expressions for $G_{l k}$ and $H_{l k}$ are listed below,

$$
\begin{aligned}
& G_{l k}=\frac{4 \pi}{2 l+1}\left\{\frac{1}{2 l+3}\left[v^{-(l+1)} M_{g l k}+v^{l+2} N_{g l k}\right]-\frac{1}{2 l-1}\left[v^{-(l-1)} S_{g l k}+v^{l} W_{g l k}\right]\right\}, \\
& H_{l k}=\frac{4 \pi}{2 l+1}\left\{\frac{1}{2 l+3}\left[v^{-(l+1)} M_{h l k}+v^{l+2} N_{h l k}\right]-\frac{1}{2 l-1}\left[v^{-(l-1)} S_{h l k}+v^{l} W_{h l k}\right]\right\} .
\end{aligned}
$$

with

$$
\begin{aligned}
& M_{g l k}=\int_{0}^{\frac{p m^{\prime}}{m}} v^{\prime l+2} p^{\prime 2} F_{l k} d p^{\prime}, N_{g l k}=\int_{\frac{p m^{\prime}}{m}}^{\infty} \frac{p^{\prime 2} F_{l k} d p^{\prime}}{v^{l+1}}, S_{g l k}=\int_{0}^{\frac{p m^{\prime}}{m}} v^{\prime l} p^{\prime 2} F_{l k} d p^{\prime}, W_{g l k}=\int_{\frac{p m^{\prime}}{m}}^{\infty} \frac{p^{\prime 2} F_{l k} d p^{\prime}}{v^{\prime l-1}} \\
& M_{h l k}=\int_{0}^{\frac{p m^{\prime}}{m}} \frac{v^{l+2} p^{\prime 2} F_{l k} d p^{\prime}}{\gamma^{\prime}}, N_{h l k}=\int_{\frac{p m^{\prime}}{m}}^{\infty} \frac{p^{\prime 2} F_{l k} d p^{\prime}}{\gamma^{\prime} v^{\prime l+1}}, S_{h l k}=\int_{0}^{\frac{p m^{\prime}}{m}} \frac{v^{\prime l} p^{\prime 2} F_{l k} d p^{\prime}}{\gamma^{\prime}}, W_{h l k}=\int_{\frac{p m^{\prime}}{m}}^{\infty} \frac{p^{\prime 2} F_{l k} d p^{\prime}}{\gamma^{\prime} v^{l-1}} .
\end{aligned}
$$

\section{CODE DEVELOPMENT AND NUMERICAL RESULTS}

Generally, it is hard to get analytical results for the above kinetic equation. Therefore, we developed the corresponding numerical code in order to understand energy deposition. Several numerical techniques are developed to deal with the involved complexity of the relativistic kinetic equation. Multi-dimensional integration algorithm with high resolution is applied to calculate the coefficients. Implicit finite volume 

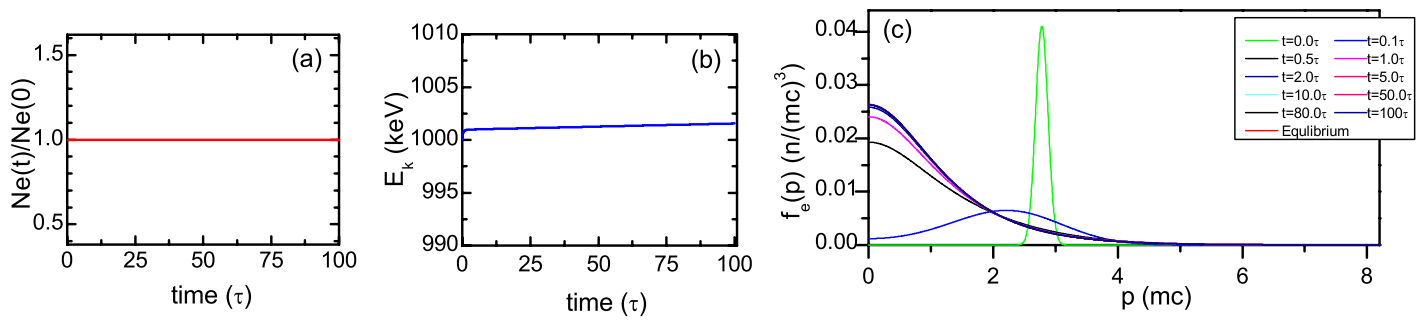

Figure 1. The evolution of $1.0 \mathrm{MeV}$ mono-energetic electron beam with self-collisions, (a) the normalized electron number, (b) the averaged kinetic energy, and (c) the normalized electron distribution function. The time is measured in unit of characteristic collision time.
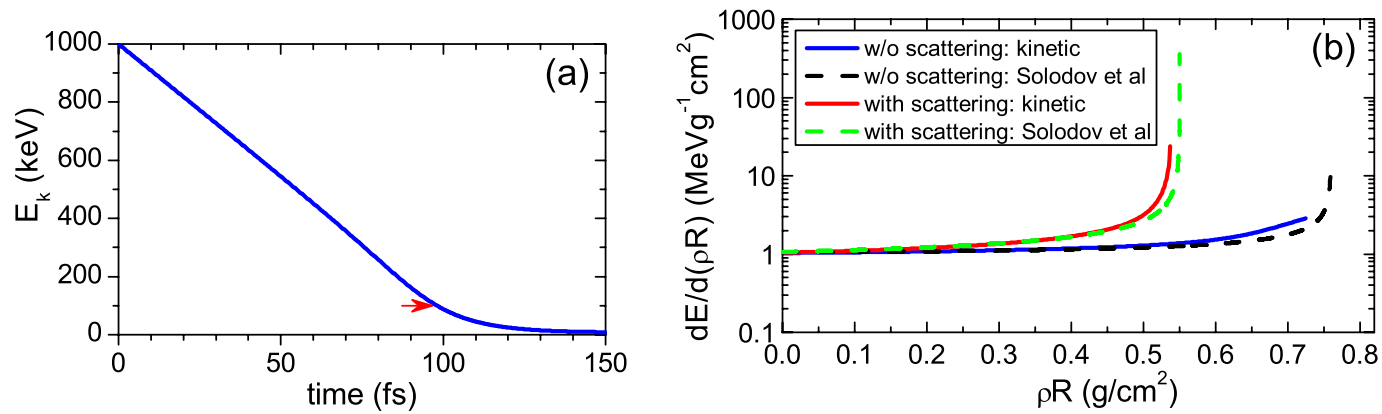

Figure 2. $1.0 \mathrm{MeV}$ mono-energetic electron beam incident into DT plasmas with mass density $\rho=300 \mathrm{~g} / \mathrm{cm}^{3}$. (a) The average beam energy. (b) The stopping power versus the electron penetration: with (red solid) and without scattering case (blue solid), which is compared with the published results in Ref. [7] (green dashed and black dashed lines, respectively).

method and nonlinear flux limiter are designed to guarantee the conservative properties of kinetic equation and non-negative distribution function. A benchmark case is showed in Fig. 1. Physically, a system with relativistic mono-energetic electrons will eventually reach equilibrium through selfcollisions without any loss of particle number and kinetic energy. The conservation is well reproduced with our code as indicated by Figs. 1(a) and (b). Fig. 1(c) shows that after about several tens of characteristic collision time, the system approaches its equilibrium state.

Fig. 2 shows a relevant energy deposition case in FI. Fig. 2(a) gives the history of electron beam energy. The red arrow shows the stopped time for the beam (with the loss of $90 \%$ initial energy [9]) is about $97.8 \mathrm{fs}$. The approximately analytical result for this case is $102.1 \mathrm{fs}$ [12], showing a reasonable agreement between the two results. The corresponding stopping power for the electron beam is plotted in Fig. 2(b) and compared with the stopping power model in Ref. [7]. It is clear that whether scattering is involved or not, the kinetic model results agree very well with these published results, demonstrating the robustness and validity of kinetic model within the context of FI. Furthermore, we change the parameter for the initial energy of fast electrons and consider the typical energy involved in realistic FI scheme. In this way, the relevant beam range and penetration depth along initial direction can be calculated, as shown in Fig. 3. The lines with squares represent the results based on kinetic model. The most recent published results by Solodov et al. [7] (lines with circles) and Atzeni et al. [9] (lines with triangles) are also displayed. It is clearly seen that the three results agree very well with each other in both continuous range and penetration depth, indicating the applicability of the kinetic code. 

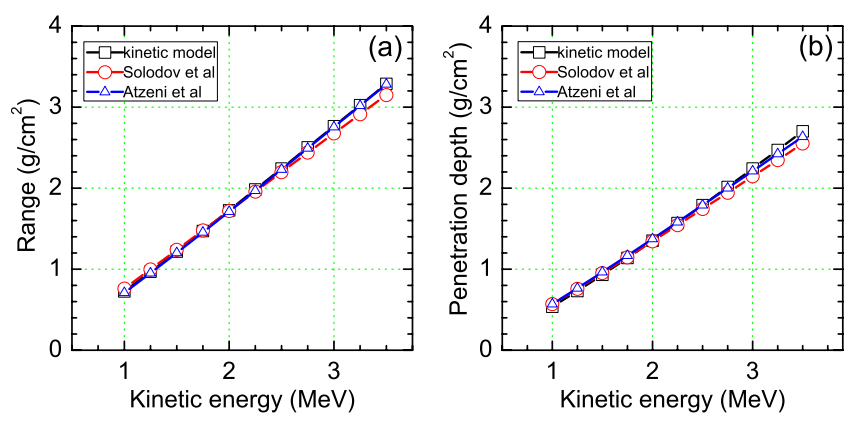

Figure 3. (a) The range and (b) the penetration depth for fast electrons with initial kinetic energy of $1 \sim 3.5 \mathrm{MeV}$ which is typically relevant to FI. The lines with squares (black), circles (red), and triangles (blue) represent the kinetic model results, the published results in Ref. [7] and Ref. [9], respectively.

\section{SUMMARY}

We have established a relativistic kinetic model for energy deposition of fast electrons in highly dense core plasmas in FI. Both binary collisions and collective response are well included. The kinetic equation is explicitly formulated in 3D momentum space by means of spherical harmonics expansion technique. The relevant numerical code is developed and verified physically. Preliminary numerical results relevant to FI agree very well with recently published works, indicating that the kinetic model and the code are of practical use in FI.

This work is supported by the National Natural Science Foundation of China (Grant Nos. 10835003, 10935003, 10974022,11175026 and 11175029).

\section{References}

[1] M. Tabak et al., Phys. Plasmas 1, 1626 (1994)

[2] C. Deutsch et al., Phys. Rev. Lett. 77, 2483 (1996)

[3] R. Kodama et al., Nature (London) 412, 798 (2001); R. Kodama et al., 418, 933 (2002)

[4] K. V. Starikov and C. Deutsch, Phys. Rev. E 71, 026407 (2005)

[5] M. H. Key, Phys. Plasmas 14, 055502 (2007)

[6] C. K. Li and R. D. Petrasso, Phys. Rev. E 73, 016402 (2006); Phys. Plasmas 13, 056314 (2006)

[7] A. A. Solodov and R. Betti, Phys. Plasmas 15, 042707 (2008)

[8] T. Yokota et al., Phys. Plasmas, 13, 022702 (2006)

[9] S. Atzeni et al., Plasma Phys. Control. Fusion 51, 015016 (2009)

[10] Yu. L. Klimontovich, Kinetic Theory of Nonideal Gases and Nonideal Plasmas (Pergamon Press, 1982)

[11] M. N. Rosenbluth et al., Phys. Rev. 107, 1 (1957)

[12] J. Robiche et al., Phys. Rev. E 70, 046405 (2004) 